\title{
ANALYSIS OF AN N/G/1 FINITE QUEUE WITH THE SUPPLEMENTARY VARIABLE METHOD
}

\author{
GYEMIN LEE and JONGWOO JEON ${ }^{1}$ \\ Seoul National University \\ Department of Statistics \\ Seoul 151-742, Korea
}

(Received April, 1997; Revised April, 1998)

\begin{abstract}
In this paper, we suggest a new approach to the analysis of an N/G/1 finite queue with the supplementary variable method. Compared to the conventional approach, our approach yields a simpler formula for the queue length distribution, which in turn gives a more efficient computational algorithm. Also, the new approach enables us to derive the joint density of the queue length and the elapsed service time.

Key words: N-process, Elapsed Service Time, Imbedded Markov Chain, Supplementary Variable Method, Schur-Banachiewicz Formula.
\end{abstract}

AMS subject classifications: $60 \mathrm{~K} 25,68 \mathrm{M} 20$.

\section{Introduction}

Very complex input flows often occur in integrated service communication systems. As an approximation to such a stream, Neuts [4] introduced the N-process. This Nprocess is analytically tractable and can appropriately represent the correlation and burstiness of the stream. Many familiar arrival processes are special cases of the Nprocess.

To investigate the performance of the service facility with finite resources, Blondia [1] considered an N/G/1 finite queue, i.e., a single server queue with $K$ waiting rooms in which customers arrive according to an N-process. For the analysis, he used the imbedded Markov chain technique upon service completion epochs. He also gave a computational algorithm for the queue length distribution of the system by using the Schur-Banachiewicz formula [3] for the inverse of the block matrices.

However, the computational algorithm suggested by Blondia [1] needs a large amount of work. This motivated us to study an N/G/1 finite queue. Our aim is to obtain a more efficient computational algorithm. To this end, we employ the supplementary variable method originated by Cox [2].

This paper is organized as follows. In Section 2, we define N-process as originally

\footnotetext{
${ }^{1}$ Supported by KOSEF, 1996.

Printed in the U.S.A. C1999 by North Atlantic Science Publishing Company
} 
introduced in Neuts [4]. Section 3 consists of the joint density of the queue length and the elapsed service time and the distribution of the queue length of the $N / G / 1$ finite queue.

\section{N-Process}

Consider a continuous-time Markov process with $m$ transient states and a single absorbing state. Then the infinitesimal generator of this Markov chain has the form

$$
Q=\left(\begin{array}{cc}
T & \boldsymbol{T}^{0} \\
\mathbf{0} & 0
\end{array}\right)
$$

where $T$ is an $m \times m$ non-singular matrix with $T_{i, i}<0, T_{i, j} \geq 0$ for $i \neq j$. The vector $\boldsymbol{T}^{0}$ is non-negative and satisfied $T e+\boldsymbol{T}^{0}=\mathbf{0}$, with $e=(1, \ldots, 1)^{t}$. Let $\left(\boldsymbol{\alpha}, \alpha_{m+1}\right)$ be a vector of initial state probabilities of the Markov process. In what follows, we shall assume that $\alpha_{m+1}=0$.

Now, construct a continuous-time process by restarting the above Markov process $Q$ instantaneously after each absorption through a multinomial trial with probability $\boldsymbol{\alpha}$ and outcomes $1, \ldots, m$. Then this process is also a Markov process with the state space $\{1,2, \ldots, m\}$ and the infinitesimal generator

$$
Q^{*}=T+T^{0} A^{0}
$$

where $T^{0}$ is an $m \times m$ matrix whose columns are all $\boldsymbol{T}^{0}$ and $A^{0}=\operatorname{diag}\left(\alpha_{1}, \ldots, \alpha_{m}\right)$. A transition from the state $i$ to the state $j$ in the Markov process $Q^{*}$, which does not involve absorptionis, will be called an $(i, j)$-transition, while the others are called $(i, j)$-renewal transition. Then the $\mathrm{N}$-process is an arrival process defined in the following way [4].

(1) During any sojourn in the state $i$, there are Poisson group arrivals of rate $\lambda_{i}$ and group size of densities $\left.P \phi_{i}(k), k \geq 0\right\}$. We shall denote $\phi_{i}^{*}(z)$ the $p . g . f$. of $\left\{\phi_{i}(k), k \geq 0\right\}$, and define $\Lambda=\operatorname{diag}\left(\lambda_{1}, \ldots, \lambda_{m}\right)$ and $\Phi(z)=\operatorname{diag}\left(\phi_{1}^{*}(z)\right.$, $\left.\ldots, \phi_{m}^{*}(z)\right)$.

(2) At $(i, j)$-renewal transitions, there are group arrivals with probability density $\left\{\Phi_{i, j}(k): k \geq 0\right\}$ whose p.g.f. is $\Psi_{i, j}(z)$. Let us denote the $m \times m$ matrix $\left(\Psi_{i, j}(z)\right)$ by $\Phi(z)$.

(3) At $(i, j)$-transitions $(i \neq j)$, there are group arrivals with probability densities $\left\{\Omega_{i, j}(k), k \geq 0\right\}$, whose p.g.f. is $\Omega_{i, j}(z)$. For notational convenience, we set $\Omega_{i, i}(z) \equiv 1$ for all $i$ and define $\left(\Omega_{i, j}(z)\right)_{1 \leq i, j \leq m}$ by $\Omega(z)$.

Define the conditional probabilities

$$
P_{i, j}(n, t)=\operatorname{Pr}(J(t)=j, N(t)=n \mid J(0)=i, N(0)=0),
$$

where $N(t)$ and $J(t)$ denote the number of arrivals during $(0, t]$ and the state of the underlying Markov process $\boldsymbol{Q}^{*}$ at time $t$, respectively. We also define conditional probability matrices $\boldsymbol{P}(n, t)=\left(P_{i, j}(n, t)\right)_{1 \leq i, j<m}, n \geq 0$. It was shown in [4] that

$$
\sum_{n=0}^{\infty} z^{n} \boldsymbol{P}(n, t)=\exp (\boldsymbol{R}(z) t), 0 \leq z \leq 1,
$$


with $\boldsymbol{R}(z)=\sum_{n=0}^{\infty} z^{n} \boldsymbol{R}_{n}$ and

$$
\begin{gathered}
\boldsymbol{R}_{0}=\boldsymbol{\Lambda} \boldsymbol{\Phi}(0)-\boldsymbol{\Lambda}+\boldsymbol{T}^{0} A^{0} \circ \boldsymbol{\Psi}(0)+\boldsymbol{T} \circ \boldsymbol{\Omega}(0) \\
\boldsymbol{R}_{n}=\boldsymbol{\Lambda} \boldsymbol{\Phi}(n)+\boldsymbol{T}^{0} A^{0} \circ \boldsymbol{\Psi}(n)+\boldsymbol{T} \circ \boldsymbol{\Omega}(n), n \geq 1,
\end{gathered}
$$

where o denotes the Schur (entrywise) product of two matrices. For the upcoming analysis, we shall assume that the matrix $\boldsymbol{R}_{0}^{-1}$ exists.

\section{Analysis of an N/G/1 Finite Queue}

In this section, we will analyze the N/G?1 finite queue with the supplementary variable method. The queue size is assumed to be $K$. When describing the $\mathrm{N}$ process, we will use the same notations as in Section 2. The successive service times are independent and identically distributed according to $H(x)$. Also the hazard rate function and the mean of $H(x)$ are denoted by $r(x)$ and $\mu$ respectively.

\subsection{Supplementary Variable Method}

Let $X(t)$ denote the number of customers in the system at time $t$. We define the elapsed service time $S(t)$ as follows: If $X(t)>0, S(t)$ denotes the amount of service already received by a customer in service. Otherwise, $S(t)$ denotes the amount of time elapsed after the last service completion. Then, the triplet $(J(t), X(t), S(t))$ is a three-dimensional Markov process with state space $\{1, \ldots, m\} \times\{0, \ldots, K\} \times[0, \infty)$.

Suppose that

$$
\pi(i, n, x) d x=\lim _{t \rightarrow \infty} \operatorname{Pr}(J(t)=i, X(t)=n, x \leq S(t)<x+d x)
$$

exists for all states and define $\pi(n, x)=(\pi(1, n, x), \ldots, \pi(m, n, x))$. Then the Kolmogorov differential equations of the joint density $\pi(n, x)$ can be written down as follows:

$$
\begin{gathered}
\frac{d}{d x} \pi(0, x)=\pi(0, x) \boldsymbol{R}_{0} \\
\frac{d}{d x} \pi(n, x)=-\pi(n, x) r(x)+\sum_{k=1}^{n} \pi(k, x) \boldsymbol{R}_{n-k}, 0<n<K, \\
\frac{d}{d x} \pi(K, x)=-\pi(K, x) r(x)+\sum_{k=1}^{K} \sum_{l=K-k}^{\infty} \pi(k, x) \boldsymbol{R}_{l} .
\end{gathered}
$$

The joint density $\pi(n, x)$ should satisfy the boundary conditions

$$
\pi(0,0)=\int_{0}^{\infty} \pi(1, x) r(x) d x
$$




$$
\begin{gathered}
\pi(n, 0)=\int_{0}^{\infty} \pi(n+1, x) r(x) d x+\int_{0}^{\infty} \pi(0, x) \boldsymbol{R}_{n} d x, 0<n<K, \\
\pi(K, 0)=\sum_{i=0}^{\infty} \int_{0}^{\infty} \pi(0, x) \boldsymbol{R}_{i} d x,
\end{gathered}
$$

and the normalization condition

$$
\sum_{n=0}^{K} \int_{0}^{\infty} \pi(n, x) d \dot{x} \boldsymbol{e}=1
$$

where $\boldsymbol{e}=(1, \ldots, 1)^{t}$.

Now, we shall find the joint density $\pi(n, x)$ of the queue length and the elapsed service time. From equation (1), we obtain

$$
\frac{d}{d x} \boldsymbol{P}(n, x)=\sum_{k=0}^{n} \boldsymbol{P}(k, x) \boldsymbol{R}_{n-k}, \quad n \geq 0 .
$$

With this and equations (2)-(8), we get

$$
\begin{gathered}
\boldsymbol{\pi}(0, x)=\boldsymbol{\pi}(0,0) \boldsymbol{P}(0, x), \\
\boldsymbol{\pi}(n, x)=\sum_{k=1}^{n} \pi(k, 0) \boldsymbol{P}(n-k, x)(1-H(x)), 0<n<K, \\
\boldsymbol{\pi}(K, x)=\sum_{k=1}^{K} \sum_{i=K-k}^{\infty} \pi(k, 0) \boldsymbol{P}(i, x)(1-H(x)) .
\end{gathered}
$$

We may also derive the above solutions by conditioning on the state of the system time $x$ back.

Before finding the coefficients $\pi(n, 0)$, we consider the embedded Markov chain $\left\{J\left(\tau_{n}\right), X\left(\tau_{n}\right)\right\}$, where $\left\{\tau_{n} n \geq 0\right\}$ is the $n^{\text {th }}$ epoch of service or idle completion. Then the transition probability matrix of $\left\{J\left(\tau_{n}\right), X\left(\tau_{n}\right)\right\}$ is

$$
\boldsymbol{Q}_{E}=\left(\begin{array}{cccccc}
\mathbf{0} & \boldsymbol{U}_{1} & \ldots & \boldsymbol{U}_{K-2} & \boldsymbol{U}_{K-1} & \sum_{n=K}^{\infty} \boldsymbol{U}_{n} \\
\boldsymbol{A}_{0} & \boldsymbol{A}_{1} & \ldots & \boldsymbol{A}_{K-2} & \sum_{n=K-1}^{\infty} \boldsymbol{A}_{n} & \mathbf{0} \\
\mathbf{0} & \boldsymbol{A}_{0} & \ldots & \boldsymbol{A}_{K-3} & \sum_{n=K-2}^{\infty} \boldsymbol{A}_{n} & \mathbf{0} \\
\ldots & \ldots & \ldots & \ldots & \ldots & \ldots \\
\mathbf{0} & \mathbf{0} & \ldots & \mathbf{0} & \sum_{n=0}^{\infty} \boldsymbol{A}_{n} & \mathbf{0}
\end{array}\right),
$$

where

$$
\boldsymbol{U}_{n}=\int_{0}^{\infty} \boldsymbol{P}(0, x) \boldsymbol{R}_{n} d x=\left[-\boldsymbol{R}_{0}^{-1}\right] \boldsymbol{R}_{n}, n \geq 1
$$




$$
\boldsymbol{A}_{n}=\int_{0}^{\infty} \boldsymbol{P}(n, x) d H(x), \quad n \geq 0 .
$$

The matrix $\boldsymbol{U}_{n}$ (or $\boldsymbol{A}_{n}$ ) is the probability that $n$ customers arrive during an idle time (or a service time).

Theorem 1: The coefficients $\pi(n, 0)$ of the joint density $\pi(n, x)$ are given by

$$
\left(\boldsymbol{\pi}(0,0), \ldots, \boldsymbol{\pi}(K, 0)=\frac{1}{\mu-x_{0}\left[\mu \boldsymbol{I}+\boldsymbol{R}^{-1}(0)\right] \boldsymbol{e}}\left(\boldsymbol{x}_{0}, \ldots, \boldsymbol{x}_{K}\right),\right.
$$

where $\left(\boldsymbol{x}_{0}, \ldots, \boldsymbol{x}_{K}\right)$ is the stationary vector of the transition probability matrix $\boldsymbol{Q}_{E} \cdot I$ is an identity matrix of size $m$.

Proof: By inserting (9)-(11) into the boundary conditions, we show that $(\pi(0,0)$, $\ldots, \boldsymbol{\pi}(K, 0))$ is a positive invariant vector of $\boldsymbol{Q}_{E}$, that is,

$$
(\boldsymbol{\pi}(0,0), \ldots, \pi(K, 0))=c\left(\boldsymbol{x}_{0}, \boldsymbol{x}_{K}\right) \text { for some constant } c>0 .
$$

Applying (9)-(11) to the normalization condition, we have

$$
\pi(\dot{0}, 0)\left[-\boldsymbol{R}_{0}^{-1}\right] \boldsymbol{e}+\mu \sum_{n=1}^{\infty} \pi(n, 0) \boldsymbol{e}=1 .
$$

Therefore, we have

$$
c=\frac{1}{\mu-x_{0}\left[\mu I+R_{0}^{-1}\right] \boldsymbol{e}}
$$

So the proof is complete.

The matrices $\boldsymbol{P}(n, x)$ and $\boldsymbol{A}_{n}$ can be efficiently evaluated by means of an iterative procedure in [3]. Therefore, we can compute $\pi(n, x)$ by deriving the stationary vector of the transition probability matrix $\boldsymbol{Q}_{E}$. As Blondia [1] did, we can also reduce the complexity of the computation for the stationary vector with the Schur-Banachiewicz formula for the inverse of block matrices.

\subsection{Queue Length Distribution}

In this subsection, we shall consider two computational algorithms to obtain the queue length distribution $\pi(n)$ using the coefficients $\pi(n, 0)$ derived in the previous subsection.

Let us define

$$
\boldsymbol{M}_{n}=\int_{0}^{\infty} \boldsymbol{P}(n, x)(1-H(x)) d x, \quad n \geq 0
$$

and let $\boldsymbol{M}(z)$ be the generating function of $\left\{\boldsymbol{M}_{n}, n \geq 0\right\}$. Then equations (9)-(10) yield

$$
\begin{gathered}
\boldsymbol{\pi}(0)=\boldsymbol{\pi}(0,0)\left[-\boldsymbol{R}_{0}^{-1}\right], \\
\boldsymbol{\pi}(n)=\sum_{k=1}^{n} \boldsymbol{\pi}(k, 0) \boldsymbol{M}_{n-k}, \quad 1 \leq n \leq K-1 .
\end{gathered}
$$


Since $\sum_{n=0}^{K} \pi(n)$ is the stationary vector $\theta$ of the underlying Markov process $\boldsymbol{Q}^{*}$, we get

$$
\pi(K)=\theta-\sum_{n=0}^{K-1} \pi(n) .
$$

Using the fact that $(\pi(0,0), \ldots, \pi(K, 0))$ is an invariant vector of the transition probability matrix $\boldsymbol{Q}_{E}$ and $\boldsymbol{A}(z)=\boldsymbol{M}(z) \boldsymbol{R}(z)+\boldsymbol{I}$, we have

$$
\begin{gathered}
\boldsymbol{\pi}(n)=\left(\sum_{k=1}^{n-1} \pi(k) \boldsymbol{R}_{n-k}=\boldsymbol{\pi}(0) \boldsymbol{R}_{n-1}\right)\left[-\boldsymbol{R}_{0}^{-1}\right] \\
-\left(\mathbf{1}_{(n \geq 2)} \boldsymbol{\pi}(n-1,0)-\boldsymbol{\pi}(n, 0)\right)\left[-\boldsymbol{R}_{0}^{-1}\right], 1 \leq n \leq K-1,
\end{gathered}
$$

where $\mathbf{1}_{(\cdot)}$ is the indicator function.

The above equation (14) for the queue length distribution has a simpler form than on derived by Blondia [1], since Blondia's formulas require additional computation of matrices $\left\{\boldsymbol{R}_{n}(s), n \geq 0\right\}$ satisfying $[\boldsymbol{R}(z)+s \boldsymbol{I}]^{-1}=\sum_{n=0}^{\infty} \boldsymbol{R}_{n}(s) z^{n}$. Consequently, we can obtain a more efficient computational algorithm for the queue length distribution.

\section{References}

[1] Blondia, C., The N/G/1 finite capacity queue, Stoch. Models 5 (1989), 273-294.

[2] Cox, D.R., The analysis of non-Markovian stochastic process by inclusion of supplementary variables, Proc. Camb. Phil. Soc. 51 (1955), 433-441.

[3] Lucantoni, D.M. and Ramaswami, V., Efficient algorithms for solving the nonlinear matrix equation arising in phase type queues, Commun. Statist.-Stoch. Model 1 (1985), 29-52.

[4] Neuts, M.F., A versatile Markovian point process, J. Appl. Prob. 16 (1979), 764-779.

[5] Ouellette, D.V., Schur complements and statistics, Linear Algebra and its Applications 36 (1981), 187-295. 


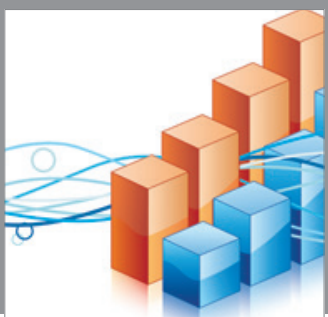

Advances in

Operations Research

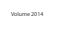

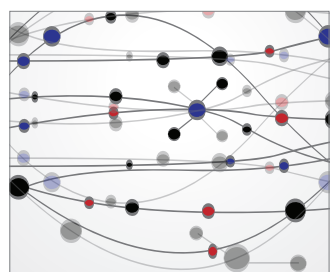

\section{The Scientific} World Journal
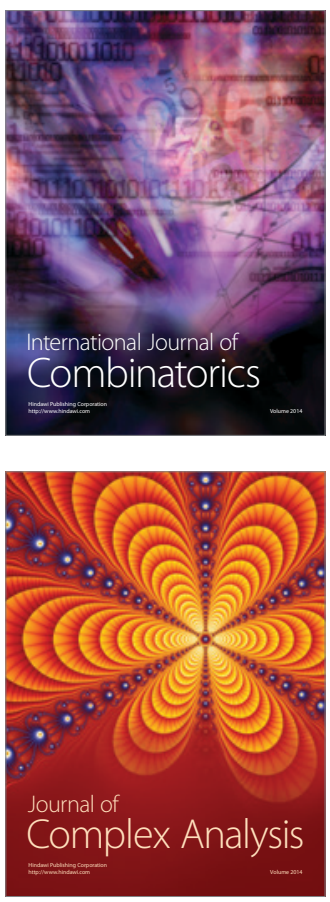

International Journal of

Mathematics and

Mathematical

Sciences
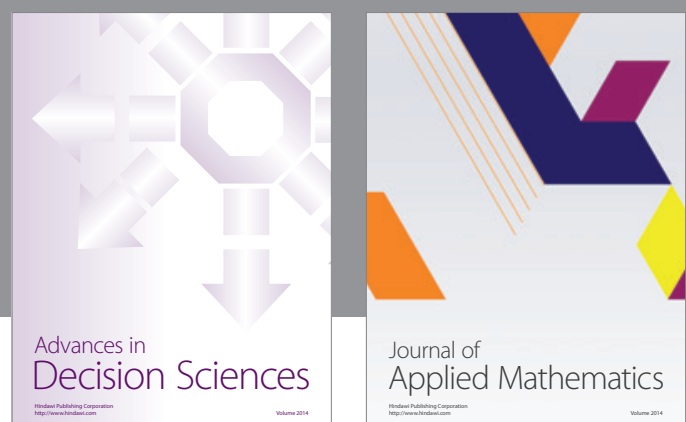

Journal of

Applied Mathematics
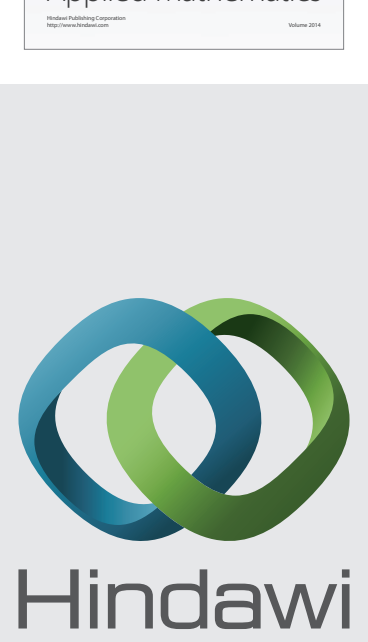

Submit your manuscripts at http://www.hindawi.com
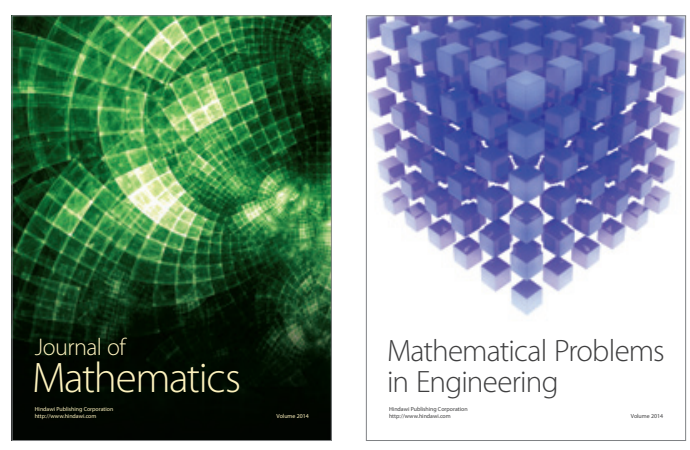

Mathematical Problems in Engineering
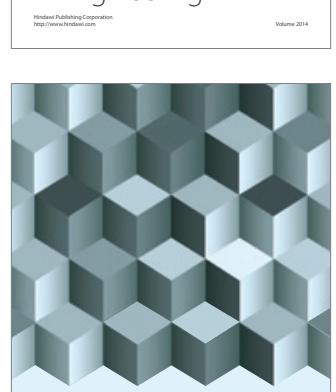

Journal of

Function Spaces
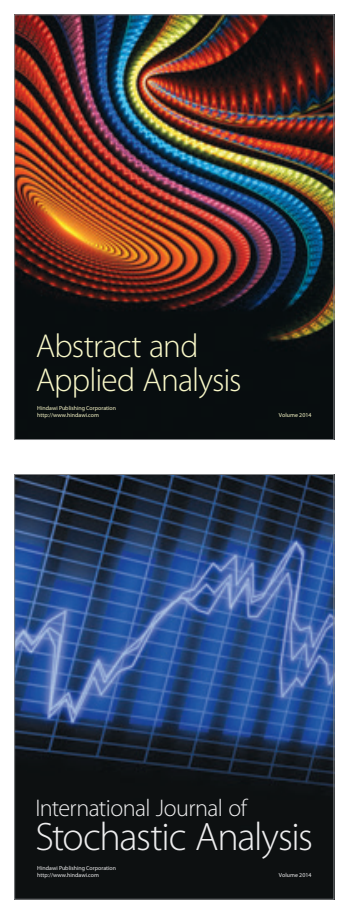

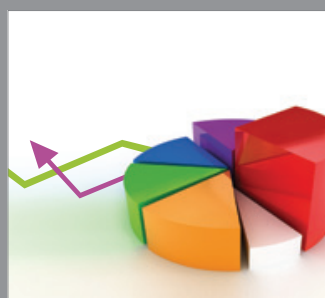

ournal of

Probability and Statistics

Promensencen
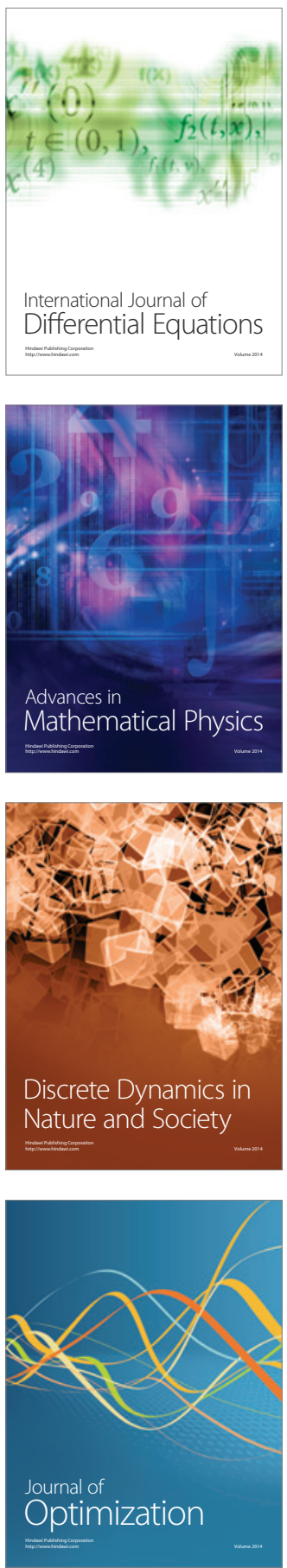